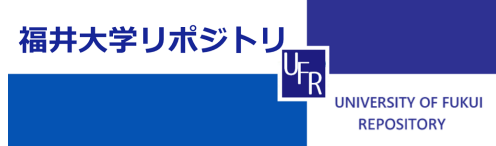

Aut omat i c Gener at i on of Fuzzy $\mathrm{Cl}$ assi $\mathrm{f} i$ cat $\mathrm{i}$ on Systens Usi ng Hyper-Cone Menber shi p Funct i ons

\begin{tabular}{|l|l|}
\hline 著者 & $\begin{array}{l}\text { HNG Li na, I NOUE H r oyuki , M YASAKA Kenj i , } \\
\text { TSUKAMOTO M t sur u }\end{array}$ \\
\hline $\begin{array}{l}\text { j our nal or } \\
\text { publ i cat i on t i t I e }\end{array}$ & $\begin{array}{l}\text { Pr oc. of 2003 I EEE I nt er nat i onal Symposi um on } \\
\text { Comput at i onal I nt el I i gence i n Robot i cs and } \\
\text { Aut omat i on }\end{array}$ \\
\hline vol une & 2 \\
\hline page r ange & 658663 \\
\hline year & 2003 \\
\hline URL & ht t p: //hdl . handl e. net /10098/1980 \\
\hline
\end{tabular}




\section{Automatic Generation of Fuzzy Classification Systems Using Hyper-Cone Membership Functions}

\author{
Lina HNG \\ Graduate School of Education \\ Fukui University \\ 3-9-1 Bunkyo, Fukui, JAPAN 910-8507 \\ linahng@centrin.net.id \\ Kenji MIYASAKA \\ Faculty of Education and Regional Studies \\ Fukui University \\ 3-9-1 Bunkyo, Fukui, JAPAN 910-8507
}

\author{
Hiroyuki INOUE \\ Faculty of Education and Regional Studies \\ Fukui University \\ 3-9-1 Bunkyo, Fukui, JAPAN 910-8507 \\ inoue@edu00.f-edu.fukui-u.ac.jp \\ Mitsuru TSUKAMOTO \\ Faculty of Education and Regional Studies \\ Fukui University \\ 3-9-1 Bunkyo, Fukui, JAPAN 910-8507
}

\begin{abstract}
In this paper, we propose automatic generation methods of fuzzy classification rules with the Genetic Algorithms (GAs) to obtain compact fuzzy systems. This time, we propose an approach of hyper-cone membership function to construct rules for the antecedent part. Then, this method is determined the location and shape of hyper-cone membership function in the antecedent part, output class and the number of necessary inputs of each rule by GAs. Also, using the rule addition method in GA process, compact fuzzy classification systems are obtained. Though the proposed methods are quite simple, the process of GAs on both methods presents a solving for two-objective optimization problems: increasing the numbers of correct pattern classification, while decreasing the rule and input numbers optimally. This method was applied to Wine data sets and Wisconsin Prognostic Breast Cancer (WPBC) data sets. Wine data sets consist of 13 inputs and three outputs, while WPBC data sets contain 33 inputs and two outputs.
\end{abstract}

\section{Kyewords}

Fuzzy Classification System, Hyper-cone Membership Function, Genetic Algorithms

\section{Introduction}

Fuzzy systems using fuzzy reasoning have been applied in various fields. However, there are tuning problems in membership functions and reasoning rules. Also, it is difficult to obtain fine fuzzy rules showing best performance for the system. Therefore, the study for automation of this processes have led to many researches with various tools for system development. For example, there are neural networks [1, 2, 3], genetic algorithms (GAs) $[4,5]$, clusterings $[6,7]$ and so on. Also, many methods of automatic generation for pattern classification problems using fuzzy systems have been proposed. Ishibuchi et al. were proposed GA based methods using various approach $[8,9,10]$. These method obtained compact fuzzy systems.

The purpose of this study is to obtain compact fuzzy classification systems dealing with high dimensional classification problems. The classification problem is a problem of estimating dimensional space and dividing the space into the regions of categories or classes. As the dimension increases, the system becomes complex.

In this paper, regarding to the classification problem, we present a method that combines fuzzy classification systems using hyper-cone membership functions $[11,12,13]$ with GAs as the searching method. The hyper-cone membership function is expressed by a kind of radial basis function, and its fuzzy rule can be flexibly located in input and output spaces. Inoue et al. have proposed GA based automatic generation techniques for fuzzy rules using hyper-cone membership functions, and applied to vehicle navigations and inverted pendulum control problems $[12,14,15]$.

We propose automatic generation methods of fuzzy classification rules using hyper-cone membership functions by GA. Dealing with high dimensional problems brings to a pre-assumption that some of the input vectors play important role in generating the rules, while others is assumed as unnecessary information. Therefore, the reduction of input number is considered to be important in this method. Also, unnecessary rules are eliminated to generate a compact fuzzy system. Therefore, to generate fuzzy classification systems, we propose an approach that refers to rule addition method. In this method, rule is added one by one, until the system reaches its best performance. The proposed method is applied to Wine Recognition Data and Wisconsin Prognostic Breast Cancer (WPBC) Data [16].

\section{Fuzzy Pattern Classification Using Hyper- Cone Membership Functions}

In $[11,12]$, fuzzy control rules using hyper-cone membership functions have been applied. In this paper, we propose fuzzy classification rules using hyper-cone membership functions. Therefore, fuzzy subsets $A_{i}$ in the antecedent part is expressed by the hyper-cone membership function. Also, concept of "don't care input" is introduced, in order to reduce the number of inputs in each rule. 


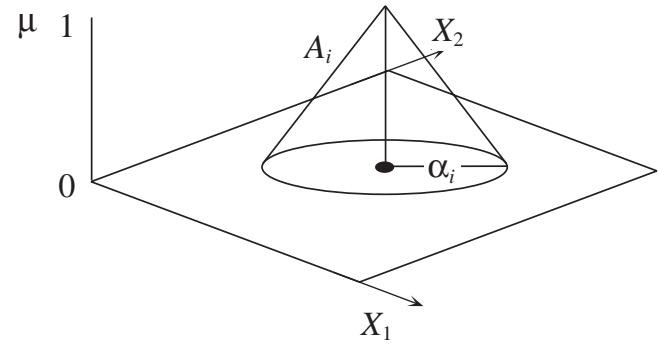

Figure 1: Shape of hyper-cone membership function $\left(l^{i}=2\right)$

\subsection{Fuzzy Classification Rules Using Hyper- Cone Membership Functions}

In this method, the fuzzy rule $R^{i}$ are expressed as follows:

$$
R^{i}: \text { if } \boldsymbol{x}_{i} \text { is } A_{i} \text { then class is } C_{i}, i=1,2, \cdots, n
$$

where $i$ is rule number, $n$ is the number of rules, $\boldsymbol{x}_{i}$ is the input vector of $R^{i}, A_{i}$ is the fuzzy subsets of the antecedent part, and $C_{i}$ is the output class. In this fuzzy system, the number of inputs (attributes) is $L$, and the input vector $\boldsymbol{x}$ for the fuzzy system is defined by Eq.(2).

$$
\boldsymbol{x}=\left[\begin{array}{llll}
x_{1} & x_{2} & \cdots & x_{L}
\end{array}\right]^{\mathrm{T}}
$$

However, it seems that there exist important rules and unnecessary rules in input vector $\boldsymbol{x}$. Therefore, fuzzy rule use only necessary attributes, and other attributes are assumed "don't care input". Therefore, the input vector of each rule $\boldsymbol{x}_{i}$ is defined by Eq.(3).

$$
\boldsymbol{x}_{i}=\left[\begin{array}{llll}
x_{i 1} & x_{i 2} & \cdots & x_{i l^{i}}
\end{array}\right]^{\mathrm{T}}
$$

where $l^{i}$ is the number of inputs of rule $R^{i}$.

The fuzzy subsets $A_{i}$ of the rule $R^{i}$ is expressed by hyper-spherical fuzzy subsets directly corresponding to a subspace in input space. Therefore, fuzzy subsets $A_{i}$ is defined by a hyper-cone membership function described below. In this fuzzy system, since there are $n$ fuzzy rules, $n$ hyper cone membership functions are located in input space.

The hyper-cone membership function $\mu_{A_{i}}\left(\boldsymbol{x}_{i}\right)$ in $l^{i}$ dimensional input space is defined by Eqs.(4) and (5).

$$
\begin{gathered}
\mu_{A_{i}}: A_{i} \rightarrow[0,1] \\
\mu_{A_{i}}\left(\boldsymbol{x}_{i}\right)=\left(1-\frac{\left\|\boldsymbol{x}_{i}-\boldsymbol{a}_{i}\right\|}{\alpha_{i}}\right) \vee 0
\end{gathered}
$$

where $\boldsymbol{a}_{i}$ and $\alpha_{i}$ are the center coordinate vector and the radius of the fuzzy subsets $A_{i}$, respectively. In Eq.(5), the center coordinate $\boldsymbol{a}_{i}$ are given by:

$$
\boldsymbol{a}_{i}=\left[\begin{array}{llll}
a_{i 1} & a_{i 2} & \cdots & a_{i l^{i}}
\end{array}\right]^{\mathrm{T}}
$$

The membership function $\mu_{A_{i}}$ has a grade 1.0 at the center $\boldsymbol{a}_{i} \in \boldsymbol{R}^{l^{i}}$ of the fuzzy subset $A_{i}$ whose radius is $\alpha_{i}$. The membership value decreases in proportion to the distance from the center $\boldsymbol{a}_{i}$. At the circumference of this sphere, a grade has 0.0. Figure 1 shows the hypercone membership function in case of $l^{i}=2$.

The use of hyper-cone membership functions does not give the limitation to the size of membership functions. Therefore, hyper-cone membership function can be flexibly used even in the high dimensional cases.

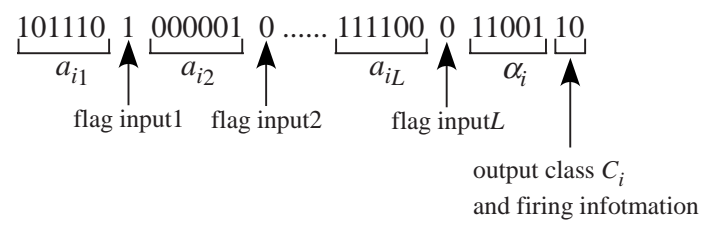

Figure 2: Chromosome of fuzzy rule $R^{i}$

\subsection{Reasoning Method}

Membership value $\mu_{A_{i}}$ of rule $R^{i}$ for input vector $\boldsymbol{x}$ is determined by Eq.(5). Therefore, the truth value $\omega_{i}$ of each fuzzy rule is calculated as follows:

$$
\omega_{i}=\mu_{A_{i}}, i=1,2, \cdots, n
$$

Output class $C_{\text {out }}$ is class $C_{i}$ having the maximum truth value $\omega_{\text {out }}$ (see Eq.(8)).

$$
\omega_{\text {out }}=\bigvee_{i=1}^{n} \omega_{i}
$$

\section{Fuzzy Classification System Generation Methods by GAs}

We generate fuzzy classification rules with hyper-cone membership functions by GAs. In this GAs process, we apply Pits approach method. In this method, one fuzzy system (a population of fuzzy rules) is made by one individual in the GAs process.

In this method, in order to obtain compact fuzzy systems for high dimensional problems, we propose a fuzzy rule generation method with the rule addition method.

\subsection{Fuzzy Rule Generation Method Using GAs}

\subsubsection{Coding of Genes}

Genetic information of fuzzy rule $R^{i}$ is the location and the shape of input membership function and the output class. Therefore, parameters of hyper-cone membership functions composed one fuzzy rule are searched by GA. Genetic parameters of fuzzy rule $R^{i}$ are as follow

- Center coordinate $\boldsymbol{a}_{i}$ of fuzzy subset $A_{i}$

- Each input flag that is "don't care input" or not,

- Radius $\alpha_{i}$ of fuzzy subset $A_{i}$

- Output class $C_{i}$ of fuzzy rule $R^{i}$, and

- Firing information.

In this information, each input flag is determined whether each input is used or not in a rule. If the flag of input $j$ in rule $R^{i}$ contains information that is considered as 'un-used' information, input data $x_{j}$ is not used to calculate the membership value of rule $R^{i}$, in other words, the input is "don't care input". Also, firing information indicates for a sign whether rule $R^{i}$ is fired or not in the fuzzy system. If this information is determined to be 'not fired', the rule $R^{i}$ is not used in fuzzy reasoning. The genes of one chromosome for rule $R^{i}$ is shown in Figure 2 .

One individual $I_{p}(p=1,2, \cdots, P)$ consists of $n$ fuzzy rules. So, one individual expresses $n$ chromosomes in one strings. The population is composed of $P$ individuals (see Figure 3 ). Then, $P$ is the population size. 


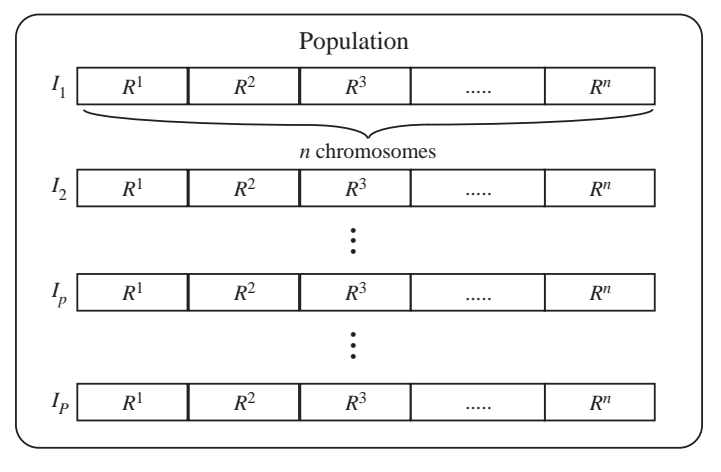

Figure 3: Composition of one population $P$

\subsubsection{Fitness Function}

In order to maximize the number of correctly classified patterns and minimize the number of fuzzy rules and inputs, the fitness $\mathrm{fit}_{p}$ for each individual is applied as Eq.(9).

$$
f i t_{p}=w_{1} c o_{p}-w_{2} r n_{p}-w_{3} i n_{p}
$$

where $c o_{p}$ is the number of correct patterns, $r n_{p}$ is the number of the rules, and $i n_{p}$ is the number of used inputs. Then, $w_{1}, w_{2}$ and $w_{3}$ represent value of weight, respectively.

\subsubsection{Genetic operation}

The procedure of genetic operations is as follows:

Step 1: Initial population is generated. Generally, this process is carried out randomly. In the method described 3.2, however, some genes are defined some determined information. Also, some genes are fixed during GA operations.

Step 2: Evaluate the fitness of each individual $I_{p}$ in the population.

Step 3: Create a new population by repeating following steps until a population size $P$ is complete.

Selection: Select two parent chromosomes from a population according to their fitness. This method applies the roulette wheel model.

Crossover: A single point crossover method is conducted to form a new offspring.

Mutation: With a mutation rate, mutate new offspring at each locus (position in chromosome).

Accepting: Place new offspring in a new population.

Step 4: Use new generated population for a further run of algorithm when the number of population reaches $P$

Step 5: . If the process reaches $G_{\text {end }}$ generation, the process stops. Otherwise return to Step 2.

\subsection{Rule Addition Method}

In this section, a rule addition method, that the fuzzy rules generation method described before section is effectively combined, is proposed. The proposed method refers to rule addition method, where rule is added one

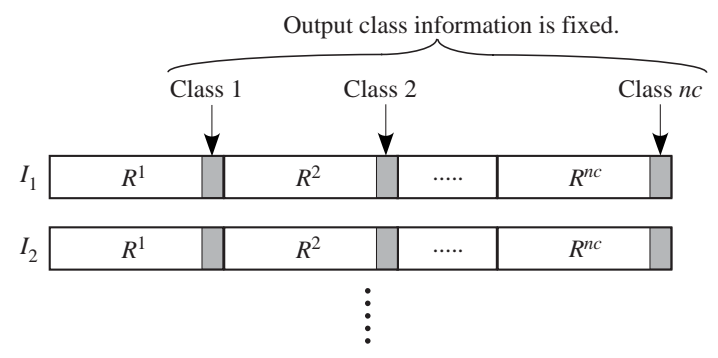

Figure 4: Composition of population in first stage

by one, until the system reaches its best performance. This method divides the process of GAs into two stages of process. Though the proposed method is quite simple, the process of GAs presents a solving for two-objective optimization problems: decrease the number of inputs and rules, and increasing the correct pattern number.

\subsubsection{First Stage}

In the first stage, minimum fuzzy rules are generated by GAs. In this stage, one fuzzy rule is generated in each class. Therefore, the number of fuzzy rules is the number of classes, and only main rule of each class is obtained by GA process.

If the number of classes is $n c$, the number of rules $n$ in this stage is $n c$. Then, fuzzy rule $R^{i}$ is the rule to determine output class $i$. So, it needs not to decide output class by GA, because output class of each rule is fixed. Therefore, only the location and shape of hypercone membership function in the antecedent part are assigned by GAs.

The genes of one individual for rule $R^{i}$ in the first stage is shown in Figure 4. In this figure, some genes expressed output class of each rule are fixed. These genes are provided class information directly, and other genes are generated randomly. This gene strings is one individual $I_{p}$, and population is composed of $P$ individuals. Though genetic operations described in 3.1 are implemented using this population, the mutation operation is not carried out for output class genes, because output class of each rule is fixed. If generation number reaches $G_{1 s t}$, genes string having maximum fitness value during all generations is defined as maximum gene string $S_{\max }$, and continue to the second stage.

In this stage, fuzzy systems, which are one rule par one class, and comparatively high correct pattern number, are obtained.

\subsubsection{Second Stage}

In this stage, each one fuzzy rule is added to fuzzy rules composed of the maximum gene string $S_{\max }$, and GA operation is executed. Therefore, genes of one fuzzy rule are randomly generated, and combined the maximum gene string $S_{\max }$.

The composition of individuals in population is shown in Figure 5. In this stage, chromosome of one individual consists that genes of $S_{\max }$ are used the first $n$ rules as it is, and genes of the $n+1$ th rule are generated randomly. This gene strings is one individual $I_{p}$, population is composed of $P$ individuals, and genetic operations described in 3.1 are implemented using this population. In initial population, the first $n$ rules of all individuals are same because of using data of $S_{\max }$. Also, genetic operations 


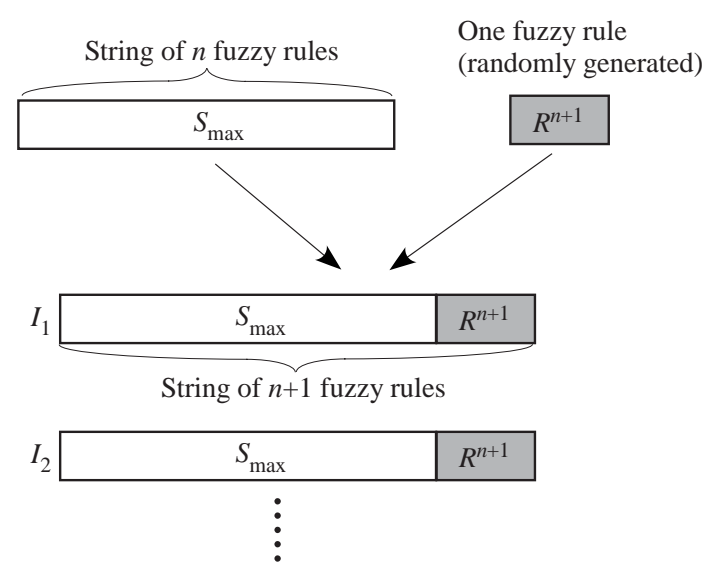

Figure 5: Composition of individuals in second stage

are carried out for all genes of each rule, though the mutation operation is not carried out for output class genes in the first stage. So, the location and shape of hyper-cone membership function in the antecedent part and the class in the consequent part of that rule are assigned by GAs.

After $G_{2 n d}$ generations, genes string having maximum fitness value during $G_{2 n d}$ generations is defined as maximum genes string in the second stage $S_{\max }^{2 n d}$. If $S_{\max }^{2 n d}$ shows better performance than $S_{\max }$, the maximum gene string $S_{\max }$ is exchanged with $S_{\max }^{2 n d}$.

This procedure are iterated to $T_{2 n d}$ times. Finally, the fuzzy rules of $S_{\max }$ is the obtained fuzzy system by GAs.

\subsubsection{Procedure of Rule Addition Method}

The procedure of the rule addition method with the first stage and the second stage is following:

[First Stage ]

Step 1: Generated initial population like Figure 4.

Step 2: Genetic operations are curried out.

Step 3: After $G_{1 s t}$ generations, genes string having maximum fitness value during $G_{1 s t}$ generations is defined as maximum gene string $S_{\max }$.

\section{[Second Stage ]}

Step 4: Initial population like Figure 5 is generated using genes of $S_{\max }$.

Step 5: Genetic operations are curried out.

Step 6: After $G_{2 n d}$ generations, genes string having maximum fitness value during $G_{2 n d}$ generations is defined as maximum gene string $S_{\max }^{2 n d}$.

Step 7: If $S_{\max }^{2 n d}$ shows better performance than $S_{\max }$, the maximum gene string $S_{\max }$ is exchanged with $S_{\max }^{2 n d}$.

Step 8: If iteration number reaches $T_{2 n d}$, then go to Step 9. Otherwise, go to Step 4.

Step 9: the fuzzy rules of $S_{\max }$ is the obtained fuzzy system by GAs.
Table 1: Setting of parameters (Wine data)

\begin{tabular}{|c|c|c|}
\hline & $\begin{array}{c}\text { Proposed } \\
\text { Method }\end{array}$ & $\begin{array}{c}\text { Comparative } \\
\text { Method }\end{array}$ \\
\hline $\begin{array}{l}\text { Generation } \\
\text { parameter }\end{array}$ & $\begin{array}{cc}G_{1 s t} & 5000 \\
G_{2 n d} & 1000 \\
T_{2 n d} & 5\end{array}$ & $G_{\text {end }}$ \\
\hline Population size $P$ & 30 & 30 \\
\hline Initial rule number & 3 & 30 \\
\hline Weight $w_{1}$ & $1.23 \times 10^{-2}$ & $1.38 \times 10^{-2}$ \\
\hline Weight $w_{2}$ & 0.0 & $4.17 \times 10^{-2}$ \\
\hline Weight $w_{3}$ & $2.85 \times 10^{-3}$ & $3.21 \times 10^{-3}$ \\
\hline
\end{tabular}

By applying this method, it does not require such a long process time as the system just focuses on the searching of two parameters: number of correct pattern and number of input. In this method, the rule number has already been fixed in the first stage. For that reason, number of genetic information that is used for one chromosome is much less. In the first stage, one chromosome is built based on the minimum rules. Therefore the process time significantly reduces as the searching just need to find the best combination between a few numbers of parameters and numbers of bits.

\section{Application to Pattern Classification Prob- lems}

The proposed method was applied to Wine Recognition Data and Wisconsin Prognostic Breast Cancer (WPBC) Data. These data are the well-known data set achieve that is taken from UCI Repository of Machine Learning Database [16].

For the comparison, the fuzzy rule generation method without the rule addition was applied to these data (comparative method). So, this is used only the GA based method described 3.1. Therefore, in initial GAs setting, many rules are randomly generated, and the number of rules is decreased by effectiveness of fitness function.

\subsection{Application to Wine Data}

Wine Data contains 178 data sets with 13 attributes (inputs) and three classes. In these data sets, we specify $50 \%$ of data sets on each class as training data, and the rest $50 \%$ of data set on each class as testing data. Consequently, 90 data sets were used as training data and the rest 88 data sets as testing data.

Simulation parameters of both methods are shown in Table 1. Also, the mutation rate was set into 0.01 . Using these parameters, ten simulations for each method were run to evaluate each system.

Table 2 shows simulation results of Wine Data. In proposed method, the average number of rules is 3.7. Also, the average number of inputs is 2.6. By using the input flag, the rest 10.40 inputs are considered as "don't care inputs". These values are same or smaller than results of the comparative method. Consequently, this method generates compact fuzzy systems consist of rules with few inputs. Also, the simulation shows that the average rate of correct pattern classification for training data reaches $98.60 \%$, and it reaches $97.70 \%$ for the testing data. Therefore, proposed method is shown better performance than the comparative method. 
Table 2: Simulation result of Wine data

\begin{tabular}{c|c|c|c}
\hline \multicolumn{2}{c}{} & Proposed Method & Comparative Method \\
\hline \multirow{2}{*}{ Training data (90) } & Average & $88.7(98.60 \%)$ & $84.8(94.22 \%)$ \\
\cline { 2 - 4 } & Maximum & $90(100.00 \%)$ & $86(95.56 \%)$ \\
\hline \multirow{2}{*}{ Test data (88) } & Average & $81.8(92.95 \%)$ & $78.3(89.00 \%)$ \\
\cline { 2 - 4 } & Maximum & $86(97.70 \%)$ & $84(95.45 \%)$ \\
\hline \multicolumn{2}{c}{ The average number of rules } & 3.7 & 3.5 \\
\hline \multicolumn{2}{c}{ The average number of inputs of each rule } & 2.6 & 3.3 \\
\hline
\end{tabular}

Table 3: Setting of parameters (Breast cancer data)

\begin{tabular}{|c|c|c|}
\hline & $\begin{array}{l}\text { Proposed } \\
\text { Method }\end{array}$ & $\begin{array}{c}\text { Comparative } \\
\text { Method }\end{array}$ \\
\hline $\begin{array}{c}\text { Generation } \\
\text { parameter }\end{array}$ & $\begin{array}{lc}G_{1 s t} & 3000 \\
G_{2 n d} & 1000 \\
T_{2 n d} & 2 \\
\end{array}$ & $G_{\text {end }}$ \\
\hline Population size $P$ & 30 & 30 \\
\hline Initial rule number & 2 & 30 \\
\hline Weight $w_{1}$ & $1.11 \times 10^{-2}$ & $1.20 \times 10^{-2}$ \\
\hline Weight $w_{2}$ & 0.0 & $3.88 \times 10^{-2}$ \\
\hline Weight $w_{3}$ & $1.09 \times 10^{-3}$ & $1.17 \times 10^{-3}$ \\
\hline
\end{tabular}

In Wine Case, as the consequent part comprises of three classes, the minimum value of rule number is three. Most of the simulations reach their best performance on the first stage, while the second stage plays important in performance improvement. Therefore, the system is avoided from further rule increment. The numbers of inputs significantly decrease. The proposed method obtains the fuzzy systems with small number of inputs in each rule.

\subsection{Application to Breast Cancer Data}

WPBC Data contains 198 data sets, 33 inputs and two classes. However, since four data sets are assumed to be missing, we eliminated those data sets from the simulation process. In these data sets, we specify $50 \%$ of data sets on each class as training data, and the rest $50 \%$ of data set on each class as testing data. Both training data and testing data on WPBC Case is set into 97 data sets.

Simulation parameters are shown in Table 3. Also, the mutation rate was set into 0.01 . Using these parameters, ten simulations for each method were run to evaluate each system.

Table 4 summarizes the simulation result of WPBC Data. As most of the best result is gained at the first stage where the number of rules in the first stage is set to be two, the simulations reached their best performance at the average of 2.00 rules. By using the input flag in the GA process, about 7.48 inputs is used in a rule. In these results, this method generates more compact fuzzy systems of WPBC data classification than the comparative method.

The simulation result shows that the average rate of correct pattern classification for training data reaches $76.29 \%$, and it reaches $76.19 \%$ for the testing data. Therefore, proposed method is shown better performance than the comparative method a little.

As the consequent part comprises of two classes, the minimum value of rule number is two. The numbers of rules can be reduced into the minimum number. The best performance of the system is found in the first stage (where rule is fixed by two).

However, about $24 \%$ of data sets fails to be covered by the rules. It seems that the minimum fuzzy systems with a certain level of accuracy are obtained by the first stage GA operations. However, in the second stage, additional rules to improve the system performance are not found. It is necessary to consider methods for adding effective rules in the second stage.

\section{Conclusion}

In this paper, we presented a new approach regarding to the high-dimensional pattern classification problems. We proposed an automatic generation method of fuzzy classification system using hyper-cone membership functions by GAs. Also, the proposed method referred to rule addition method. In this method, rule was added gradually until the best performance was achieved. This method is applied to classification problems of Wine Data and WPBC Data. In simulation results of Wine Data, effective and compact fuzzy systems were obtained. Also, in the WPBC Data, simulation results of presented method were shown better performance than the comparative method. However, it is necessary to consider methods for adding effective rules in the second stage.

In the future work, we would like to improve the feasibility of the fuzzy systems using the GAs to simulate some parameters. Also, the combination of hyper-cone membership function with other searching method, as Neural Network, or combination both GAs and Neural Network is considered. The purpose is to find the best combination among number of the correct pattern, number of rule and number of input to perform maximum result.

\section{References}

[1] S. Horikawa, T. Furuhashi, and Y. Uchikawa. On fuzzy modeling using fuzzy neural networks with the back-propagation algorithm. IEEE Transactions of Neural Networks, 3(5):801-806, 1992.

[2] I. Hayashi, H. Nomura, H. Yamasaki, and N. Wakami. International journal of approximate reasoning. Construction of Fuzzy Inference rules by NDF and NDFL, 6(2):241-266, 1992.

[3] J. A. Dickerson and B. Kosko. Fuzzy function approximation with ellipsoid rules. IEEE Transactions of Systems, Man, Cybernetics-B, 26(4):542560, 1996 .

[4] M. A. Lee and H. Takagi. Integrating design stage of fuzzy systems using genetic algorithms. In Proceedings of 2nd IEEE International Conference on on Fuzzy Systems, pages 612-617, 1993. 
Table 4: Simulation result of WPBC data

\begin{tabular}{c|c|c|c}
\hline \multicolumn{2}{l}{ Training data (97) } & Proposed Method & Comparative Method \\
\cline { 2 - 4 } & Average & $74.0(76.39 \%)$ & $73.5(75.77 \%)$ \\
\hline \multirow{2}{*}{ Test data (97) } & Maximum & $75(77.32 \%)$ & $74(76.29 \%)$ \\
\cline { 2 - 4 } & Average & $73.9(76.19 \%)$ & $71.0(73.20 \%)$ \\
\hline \multicolumn{2}{c}{ The average number of rules } & $74(76.29 \%)$ & $73(75.26 \%)$ \\
\hline The average number of inputs of each rule & 2.0 & 3.7 \\
\hline
\end{tabular}

[5] K. Shimojima, T. Fukuda, and Y. Hasegawa. Rbf-fuzzy system with ga based unsupervised/supervised learning method. In Proceedings of 4 th IEEE International Conference on on Fuzzy Systems, pages 253-258, 1995.

[6] K. Kamei. An application of fuzzy clustering to controller design. Journal of Japan Society for Fuzzy Theory and Systems, 8(3):448-455, June 1996 (in Japanese).

[7] M. Ryoke, H. Tamura, and Y. Nakamori. Fuzzy rule generation by hyperellipsoidal clustering. In Methodologies for the Conception, Design and Application of Intelligent Systems (World Scientific), pages 86-89, Iizuka, Japan, October 1996.

[8] H. Ishibuchi, K. Nozaki, N. Yamamoto, and H. Tanaka. Construction of fuzzy classification systems with rectangular fuzzy rules using genetic algorithms. Fuzzy Sets and Systems, 65(2), 1994.

[9] T.Murata, H.Ishibuchi, and M.Gen. Adjusting fuzzy partitions by genetic algorithms and histograms for pattern classification problems. In Proceeding of 1998 IEEE International Conference on Evolutionary Computation, pages 9-14, Anchorage, USA, May 1998.

[10] T.Nakashima, H.Ishibuchi, and T.Murata. Evolutionary algorithms for constructing linguistic rulebased systems for high-dimensional pattern classification problems. In Proceeding of 1998 IEEE International Conference on Evolutionary Computation, pages 752-757, Anchorage, USA, May 1998.

[11] H. Inoue, K. Kamei, and K. Inoue. Auto-generation of fuzzy production rules using hyper-cone membership function by genetic algorithm. In Fuzzy Logic for the Applications to Complex Systems (World Scientific), pages 53-58, Taipei, December 1995.

[12] H. Inoue, K. Kamei, and K. Inoue. Automatic generation of fuzzy if-then rules using genetic algorithms and hyper-cone membership functions. Japanese Journal of Fuzzy Theory and Systems, 8(6):957-975, December 1996.

[13] H. Inoue, K. Kamei, and K. Inoue. Automatic generation of fuzzy rules using hyper elliptic cone membership functions by genetic algorithms. Journal of Intelligent and Fuzzy Systems, 6(1):65-81, July 1998.

[14] H. Inoue, K. Hatase, and K. Kamei. Fuzzy classifier system using hyper-cone membership functions and rule reduction techniques. In Proceedings of The 10th IEEE International Conference on Fuzzy Systems, pages 1436-1439, Melbourne, December 2001.
[15] H. Inoue, K.Matsuo, K.Hatase, K.Kamei, M.Tsukamoto, and K.Miyasaka. A fuzzy classifier system using hyper-cone membership functions and its application to inverted pendulum control. In Proceeding of 2002 IEEE Conference on Systems, $M A N$ and Cybernetics (CD ROM), page Paper Number WA2D3, Hammamet, Tunisia, October 2002 .

[16] [UCI Repository of Machine Learning Databases Network Document]. Available: ftp://ftp.ics.uci.edu/pub/machine-learningdatabases/. 